\title{
Conservative treatment for late-onset b
after trabeculectomy with mitomycin $C$ in patients with ocular surface disease
}

This article was published in the following Dove Press journal:

Clinical Ophthalmology

7 August 2012

Number of times this article has been viewed

\author{
Hideto Sagara ${ }^{1,2}$ \\ Tomohiro lida ${ }^{2,3}$ \\ Kimimori Saito ${ }^{4}$ \\ Hiroki Noji ${ }^{2}$ \\ Masashi Ogasawara ${ }^{2}$ \\ Hiroshi Oyamada ${ }^{2}$ \\ 'The Marui Eye Clinic, Fukushima, \\ ${ }^{2}$ Department of Ophthalmology, \\ Fukushima Medical University School \\ of Medicine, Fukushima, ${ }^{3}$ Tokyo \\ Women's Medical University, Tokyo, \\ ${ }^{4}$ Matuki Eye Clinic, Fukushima, Japan
}

Correspondence: Hideto Sagara Marui Eye Clinic, I-83-3, Honjinmae Haramachi-ku, Minamisoma City, Fukushima Prefecture 975-0062, Japan

$\mathrm{Tel}+8 \mid 244240101$

Fax +8 I 244240148

Email hide1234@ruby.ocn.ne.jp
Background: Sodium hyaluronate and autologous serum eye drops are used to treat ocular surface disease (OSD) and are reported to prevent and treat late-onset bleb leaks following trabeculectomy with mitomycin C. In this study, we evaluated the efficacy of a combination of sodium hyaluronate and autologous serum eye drops and treatment for obstructive meibomian gland dysfunction as a therapy for late-onset bleb leaks after trabeculectomy with mitomycin $\mathrm{C}$.

Methods: This was a retrospective, interventional, nonsimultaneous study of 12 subjects (12 eyes) of mean age of $64.3 \pm 18.3$ years with OSD and apparent late-onset bleb leaks following trabeculectomy with mitomycin C between 1998 and 2008. We compared patients diagnosed with leakages before July 2005, who had been treated with separate eye drop solutions containing $0.1 \%$ sodium hyaluronate, $50 \%$ autologous serum, and $0.3 \%$ ofloxacin (sodium hyaluronate and autologous serum group, $\mathrm{n}=7$ ), with patients diagnosed from August 2005 to December 2008, who were treated with a combination of eye drops $(0.1 \%$ sodium hyaluronate, $50 \%$ autologous serum, and $0.08 \%$ levofloxacin hydrate) and eyelid massage and warm compresses for obstructive meibomian gland dysfunction (combination eye drop group, $\mathrm{n}=5$ ).

Results: Leakage was resolved in one patient (14.3\%) in the separately treated sodium hyaluronate and autologous serum eye drop group and in five patients $(100 \%)$ in the combination eye drop group $(P=0.015)$. The period after resolution of leakage with conservative treatment was 23 months in the one eye in the sodium hyaluronate and autologous serum group and 36-61 (mean $52.4 \pm 10.1$ ) months in the five eyes in the combination eye drop group.

Conclusion: Late-onset bleb leaks following trabeculectomy with mitomycin $\mathrm{C}$ can be treated effectively using a combination of sodium hyaluronate and autologous serum eye drops, eyelid massage, and warm compresses. Furthermore, combining eye drops may improve patient adherence to the drug regimen by decreasing the frequency of administration.

Keywords: ocular surface disease, bleb leak, trabeculectomy, sodium hyaluronate, autologous serum, meibomian gland dysfunction

\section{Introduction}

Since the introduction of 5-fluorouracil and mitomycin C for glaucoma-filtering surgery, the incidence of late-onset filtering bleb leaks has increased. ${ }^{1,2}$ Filtering bleb leaks can cause hypotony maculopathy, choroidal detachment, blebitis, and endophthalmitis. ${ }^{2-4}$ Defects in the conjunctival epithelium covering the bleb wall are precursors to apparent late-onset bleb leaks. ${ }^{5}$ If these defects remain untreated, then transconjunctival aqueous egress, referred to as "oozing", occurs and may later deteriorate into point leaks. If the point leaks remain untreated, then profuse leakage may occur. We previously reported that conjunctival epithelial defects in the filtering bleb wall that occur 
after trabeculectomy with mitomycin $\mathrm{C}$ can be treated with sodium hyaluronate eye drops. Furthermore, this treatment may prevent subsequent occurrence of apparent bleb leaks. ${ }^{5}$ A recent study reported that autologous serum eye drops are not very effective for treatment of apparent bleb leaks, but are effective for treatment of oozing. ${ }^{6}$ Sodium hyaluronate and autologous serum eye drops are both used to treat ocular surface disease (OSD). ${ }^{7}$ Indeed, OSD may contribute to the occurrence of late-onset bleb leaks following trabeculectomy with mitomycin C. ${ }^{5,8}$

The tear film consists of an aqueous layer and a lipid layer. ${ }^{9}$ The aqueous layer is augmented by sodium hyaluronate ${ }^{10}$ and autologous serum eye drops. ${ }^{6,7}$ The lipids that form the lipid layer are secreted by the meibomian gland. The most common cause of meibomian gland hyposecretion is obstructive meibomian gland dysfunction. ${ }^{11}$ It is recommended that severe OSD be treated with a combination therapy of eye drops, as mentioned above, and treatments for obstructive meibomian gland dysfunction. ${ }^{7}$ Therefore, we hypothesized that this same combination therapy may be effective for treating severe late-onset bleb leaks. In the present study, we attempted to treat several cases of OSD and severe late-onset bleb leaks by combining sodium hyaluronate and autologous serum eye drops with treatments for obstructive meibomian gland dysfunction.

\section{Materials and methods}

\section{Patients and treatments}

This clinical study was retrospective, interventional, and nonsimultaneous, and examined a total of 12 eyes in 12 patients (six men and six women, mean age $64.3 \pm 18.3$ years) diagnosed with OSD and either late-onset profuse bleb leaks or late-onset bleb leaks with apparent leakage points ${ }^{5,6}$ following trabeculectomy with mitomycin C. The patients were diagnosed at either Fukushima Medical University or the Marui Eye Clinic between June 1998 and December 2008. The inclusion criteria for the study were diagnosis of OSD and either late-onset profuse bleb leaks or late-onset bleb leaks with apparent leakage points. Late-onset bleb leaks were defined as leakage with a visible aqueous stream of the bleb wall that began more than 6 months after trabeculectomy with mitomycin C. Profuse bleb leaks were defined as profuse, apparent bleb leaks with difficult to detect leak points due to the profuse aqueous stream. Exclusion criteria included an inability to understand the trial procedure and, thus, the inability to provide informed consent. The institutional review board/ethics committee of Fukushima Medical University approved this study, and all patients provided their written informed consent.
Seven patients (one man and six women, mean age $60.1 \pm 21.4$ years) were diagnosed with OSD and late-onset bleb leaks before July 2005 and were treated with $0.1 \%$ sodium hyaluronate (Hyalein ${ }^{\circledR} 0.1 \%$, Santen Pharmaceutical Co, Ltd, Osaka, Japan) and 50\% autologous serum eye drops (sodium hyaluronate and autologous serum group). These drops were administered six times daily. These patients were also simultaneously administered $0.3 \%$ ofloxacin eye drops (Tarivid ${ }^{\circledR} 0.3 \%$, Santen) four times daily to prevent bleb infections. This conservative treatment with eye drops was continued for at least 4 months. Patients who showed resolution of bleb leakages were then switched to $0.1 \%$ sodium hyaluronate only eye drops (Hyalein $0.1 \%$ ) six times daily. This revised treatment continued until the final follow-up examination. If the bleb leakages did not resolve at 4 months, then patients underwent surgical bleb revision. This first involved surgical excision of the scarred cystic conjunctiva surrounding the leaking bleb. In the second step of the revision, the relatively uninvolved conjunctiva was mobilized using a large relaxing incision in the superior fornix and then sutured over Tenon's fascia in the area of filtration. ${ }^{12}$

Five patients (all male, mean age $70.0 \pm 12.8$ years) were diagnosed with OSD and bleb leakages after July 2005 and were treated with combination eye drops (containing $0.1 \%$ sodium hyaluronate, $50 \%$ autologous serum, and $0.08 \%$ levofloxacin hydrate) along with eyelid massage ${ }^{13}$ and warm compresses $^{14}$ for obstructive meibomian gland dysfunction (combination eye drop group). The combination eye drops were administered six times a day for 4 months. Thereafter, $0.1 \%$ sodium hyaluronate eye drops (Hyalein $0.1 \%$ ) were administered six times daily until the final follow-up examination. A clinician performed eyelid massage once or twice each month. The eyelid massages used meibomian gland compressors (Yoshitomi meibomian gland compressor, PMS GmbH, Tuttlingen, Germany) to clamp the eyelids without compressing the blebs. Topical anesthetic eye drops containing $0.4 \%$ oxybuprocaine hydrochloride (Benoxil ${ }^{\circledR}$, Santen) were instilled prior to clamping. Patients were instructed to apply warm compresses (hot moist towels at approximately $40^{\circ} \mathrm{C}$ ) to their eyelids for 5 minutes twice each day. ${ }^{14}$ Eyelid massage and application of warm compresses was performed from the start of treatment until the final follow-up examination.

Intraocular pressure was measured using a noncontact tonometer (Tonoref ${ }^{\circledR}$, Nidek Co, Ltd, Aichi, Japan). History of contact lens and anti-glaucoma eye drop use was determined in all patients. Patients were advised to administer the eye drops while in a supine position so that the drops 
would permeate the upper bleb. Patient adherence to the eye drop and warm eyelid compress regimens was confirmed during each visit. Patients were instructed to contact the attending doctor as soon as possible if they experienced symptoms of bleb infection, such as decreased vision, eye pain, or conjunctival hyperemia (red eye).

\section{Diagnosis of OSD and bleb leaks}

To prevent bleb damage, moistened fluorescein strips were gently applied to the palpebral conjunctiva of the lower eyelids. Fluorescein-stained corneas and conjunctiva were then examined with a fundus camera (TRC-50LX, Topcon, Tokyo, Japan) using fluorescein angiogram mode to observe the condition of the ocular surface more clearly (Figures 1 and 2). The tear film break-up time was measured upon detection of leakage. The break-up time was measured

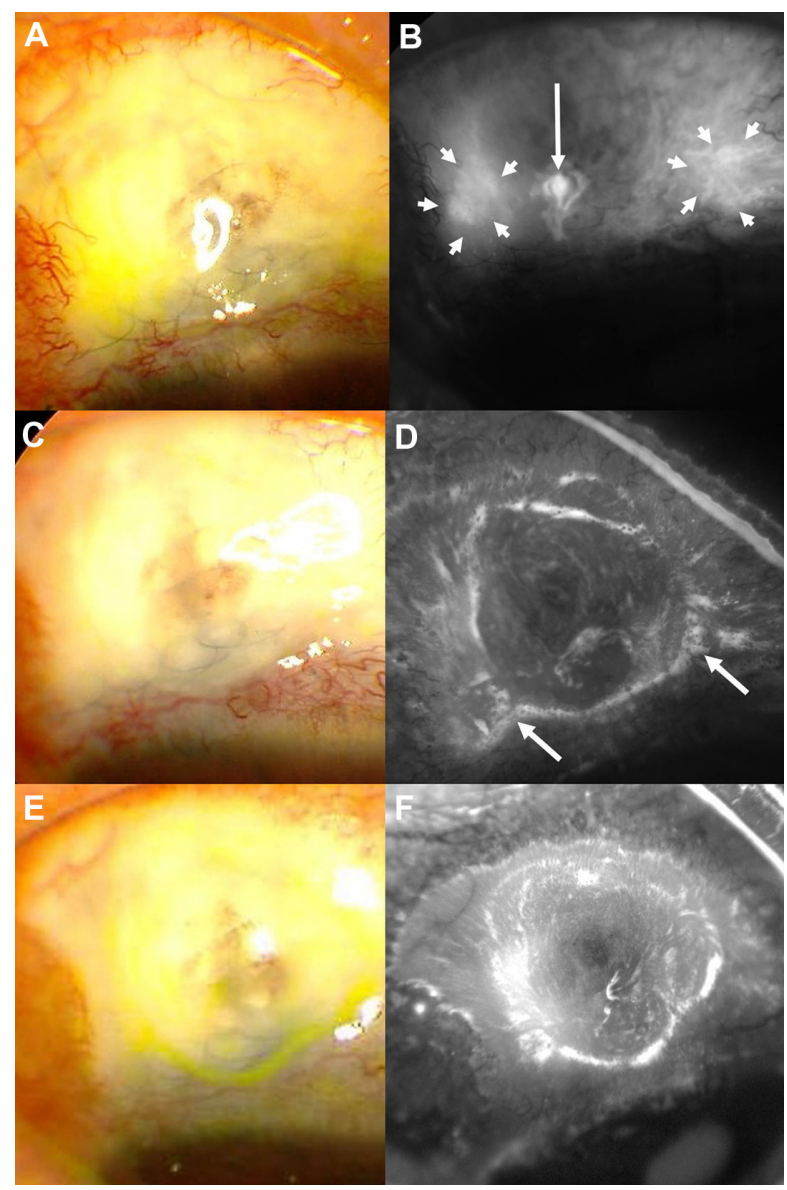

Figure I Anterior ocular segment from an 80-year-old man in the combination eye drop group photographed with a fundus camera after fluorescein staining. (A and B) Epithelial defects (arrowheads) and bleb leakage with a leak point (arrow) are visible in this monochrome photograph taken in fluorescein angiogram mode. (C and D) Four months after the start of treatment, the epithelial defects and leakage had resolved, and several microcysts were observed on the bleb wall (arrows). (E and F) By 40 months, the bleb had contracted slightly, and the number of microcysts had decreased.

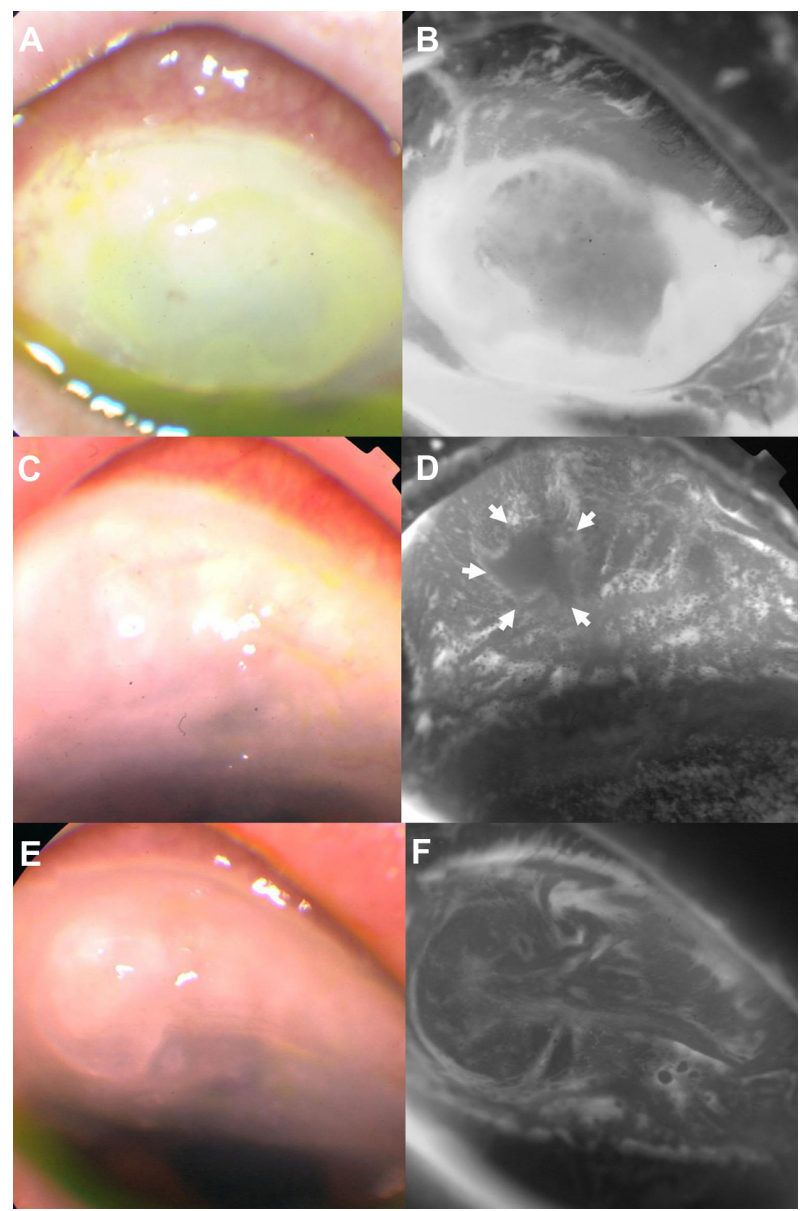

Figure 2 An 84-year-old man in the combination eye drop group. ( $\mathbf{A}$ and $\mathbf{B}$ ) Epithelial defects and profuse leakage are clearly visible. The leak point is unclear because of the profuse aqueous stream. (C and $\mathbf{D}$ ) One month after cessation of treatment with the combination eye drops, the leakage (arrowheads) recurred due to a small point leak, and many microcysts were observed on the bleb wall. Treatment with combination eye drops was recommenced. (E and F) Thirty months after recommencement of treatment, the bleb had contracted slightly and the number of microcysts had decreased.

again during the final examination of the combination eye drops group to evaluate the efficacy of eyelid massage and warm compresses. Each measurement was performed three times and a mean value was obtained. After the bleb leaks had resolved, the Schirmer test was performed without anesthesia. Ocular surface staining was evaluated according to the Oxford grading scale, ranging from grade 0 to grade $5 .^{15}$ Patients were diagnosed as abnormal when the ocular surface staining was $>$ grade 1 . Furthermore, patients were diagnosed as abnormal when the break-up time was $\leq 5$ seconds and the Schirmer score was $\leq 5 \mathrm{~mm}$, and were suspected to be abnormal if the break-up time was $<10$ seconds and the Schirmer score was $<10 \mathrm{~mm} .{ }^{16}$

Assessment of subjective symptoms and the degree of ease of induction of meibomian secretion are regarded as effective methods for diagnosing obstructive meibomian 
gland dysfunction. ${ }^{17}$ However, post-trabeculectomy bleb formations cause ocular discomfort, ${ }^{18}$ and it is then difficult to avoid compressing the blebs when applying digital pressure to the tarsus to induce meibomian secretion. Therefore, obstructive meibomian gland dysfunction could not be diagnosed in the sodium hyaluronate and autologous serum group. While meibomian gland loss can be measured without compressing the brittle blebs by using a digital ophthalmoscope (NIDEK F10 digital ophthalmoscope) in infrared reflectometry mode with an anterior segment adapter (Figure 3), this procedure was not developed until after 2005. Therefore, obstructive meibomian gland dysfunction was diagnosed by assessing lid margin abnormalities and meibomian gland $\operatorname{loss}^{17}$ in the combination eye drop group only. Four lid margin abnormalities (irregular lid margins, vascular engorgement, plugging of meibomian gland orifices, and either anterior or posterior replacement of the mucocutaneous junction) were used to assign a score from 0 to 4 based on the number of abnormalities present in each eye. ${ }^{17}$ The degree of meibomian gland loss in the upper and lower eyelids (meiboscore) ${ }^{17}$ was scored as follows: 0 (no loss); 1 (less than one-third); 2 (between one-third and two-thirds); and 3 (more than two-thirds). Lid margin abnormality scores of $\geq 2$ and summed upper and lower eyelid meiboscores of $\geq 3$ were considered abnormal.

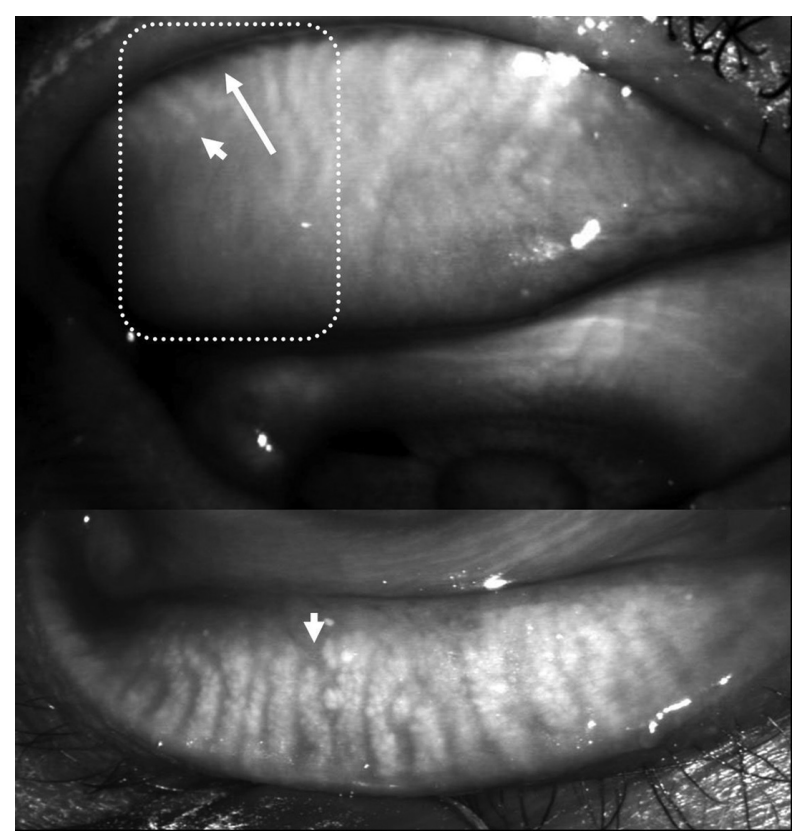

Figure 3 Image of the meibomian gland from a 74-year-old man, captured using a digital ophthalmoscope with eyelids inverted. Meibomian gland dropout (arrow) and shortening (arrowheads) can be observed.

Notes: Meibomian gland loss is particularly apparent in the areas that are in contact with the filtering bleb (encircled with dotted white lines). The meiboscore was 2 for the upper eyelid and I for the lower eyelid, giving a summed meiboscore of 3 .

\section{Preparation of autologous serum and combination eye drop solutions}

The autologous serum used in this study was prepared by collecting a blood sample of approximately $40 \mathrm{~mL}$ from each patient's antebrachial vein, which was then centrifuged for 15 minutes at $3000 \mathrm{rpm}$ under sterile conditions. The $50 \%$ autologous serum eye drops were prepared by diluting the prepared autologous serum with physiological saline (1:2). A 9.6 mL combination eye drop solution containing $0.1 \%$ sodium hyaluronate, $50 \%$ autologous serum, and $0.08 \%$ levofloxacin hydrate was prepared by mixing $3.2 \mathrm{~mL}$ of $0.3 \%$ sodium hyaluronate (Hyalein Mini 0.3\%), $4.8 \mathrm{~mL}$ of the autologous serum, and $1.6 \mathrm{~mL}$ of $0.5 \%$ levofloxacin hydrate (Cravit $0.5 \%$ ). Due to the limited shelf life of this solution, patients were instructed to discontinue use after 2 weeks.

\section{Statistical analysis}

Group differences between continuous variables were assessed using the Student's $t$-test. Differences between categorical variables were compared using either the Chisquared test or Fisher's Exact test. Differences between the scores were evaluated using Wilcoxon's rank sum test or Wilcoxon's signed-rank test. A $P$ value $<0.05$ was considered to be statistically significant. All statistical analyses were performed using JMP- ${ }^{\circledR}$ (SAS Institute, Cary, NC).

\section{Results}

Patients in the sodium hyaluronate and autologous serum group and in the combination eye drop group did not differ significantly in terms of age, diagnosis, duration of follow-up, break-up time upon detection of leakages, or Schirmer test results. The number of women in the sodium hyaluronate and autologous serum group was significantly higher than that in the combination group (Table 1). No patient had a history of contact lens use. All patients had been treated with anti-glaucoma eye drops containing benzalkonium chloride $(0.001 \%-0.02 \%)$ as a preservative for more than one year. One patient from the combination eye drop group had profuse bleb leaks, and another patient had bleb leaks with apparent leak points. Bleb leaks were resolved in only one patient (a 34-year-old woman) in the sodium hyaluronate and autologous serum group. Bleb leakage in this patient was relatively mild compared with that in other patients, and the time interval between the start of treatment and resolution of the bleb leaks was 4 months. Bleb leakages were resolved in five patients in the combination eye drop group, with a mean resolution time of $3.2 \pm 1.3$ (range 2-5) months. The time period between leakage arrest with conservative treatment and the 
Table I Demographic and clinical characteristics of the patients

\begin{tabular}{|c|c|c|c|c|}
\hline & $\begin{array}{l}\text { All patients } \\
\text { (both groups) }\end{array}$ & $\begin{array}{l}\text { SH and } \\
\text { AS group }\end{array}$ & $\begin{array}{l}\text { Combination } \\
\text { eye drop group }\end{array}$ & $P$ value \\
\hline Men/women & $6 / 6$ & $1 / 6$ & $5 / 0$ & $0.015^{\mathrm{a}}$ \\
\hline Age (years) ${ }^{b}$ & $64.3 \pm 18.3$ & $60.1 \pm 21.4$ & $70.0 \pm 12.8$ & $0.344^{c}$ \\
\hline Bleb leaks with apparent leak points/profuse leakage ${ }^{d}$ & $1 \mathrm{I} / \mathrm{I}$ & $7 / 0$ & $4 / 1$ & $0.417^{\mathrm{a}}$ \\
\hline Diagnosis & & & & $0.117^{\mathrm{a}}$ \\
\hline Primary open-angle glaucoma & 8 & 3 & 5 & \\
\hline Secondary glaucoma & 3 & 3 & 0 & \\
\hline Developmental glaucoma & I & 1 & 0 & \\
\hline Bleb leakages stopped/continued & $6 / 6$ & $1 / 6$ & $5 / 0$ & $0.015^{\mathrm{a}}$ \\
\hline \multicolumn{5}{|l|}{ Duration of follow-up (months) } \\
\hline From operation to detection of bleb leaks ${ }^{b}$ & $42.7 \pm 45.5$ & $27.4 \pm 23.4$ & $64.0 \pm 62.4$ & $0.269^{c}$ \\
\hline From resolution of bleb leaks to last examination ${ }^{b}$ & $51.8 \pm 14.7$ & $51.4 \pm 18.0$ & $52.4 \pm 10.1$ & $0.908^{c}$ \\
\hline \multicolumn{5}{|l|}{ Intraocular pressure (mmHg) } \\
\hline At time of the detected bleb leaks ${ }^{b}$ & $8.6 \pm 4.3$ & $8.7 \pm 3.1$ & $8.4 \pm 6.1$ & $0.919^{c}$ \\
\hline During last examination ${ }^{\mathrm{b}}$ & $12.6 \pm 6.5$ & $13.9 \pm 7.2$ & $10.8 \pm 5.6$ & $0.449^{c}$ \\
\hline Tear film break-up time (seconds) ${ }^{b}$ & $5.7 \pm 2.7$ & $6.6 \pm 2.8$ & $4.4 \pm 2.1$ & $0.176^{c}$ \\
\hline Range of break-up-times $(\leq 5 \mathrm{sec} / 5<<10 \mathrm{sec} / \geq 10 \mathrm{sec}$ ) & $6 / 4 / 2$ & $3 / 2 / 2$ & $3 / 2 / 0$ & $0.424^{\mathrm{a}}$ \\
\hline Schirmer test $(\mathrm{mm})^{\mathrm{b}}$ & $8.9 \pm 6.1$ & $9.1 \pm 5.8$ & $8.4 \pm 6.4$ & $0.838^{c}$ \\
\hline Range of Schirmer test $(\leq 5 \mathrm{~mm} / 5<<10 \mathrm{~mm} / \geq 10 \mathrm{~mm})$ & $4 / 1 / 7$ & $2 / 1 / 4$ & $2 / 0 / 3$ & $0.660^{\mathrm{a}}$ \\
\hline \multicolumn{5}{|l|}{ Ocular surface staining using the Oxford grading scale ${ }^{b}$} \\
\hline before treatment & $3.1 \pm 1.2$ & $3.4 \pm 1.1$ & $2.8 \pm 1.3$ & $0.394^{\mathrm{e}}$ \\
\hline last examination & $1.4 \pm 1.2$ & $2.0 \pm 1.2$ & $0.6 \pm 0.9^{*}$ & $0.065^{\mathrm{e}}$ \\
\hline
\end{tabular}

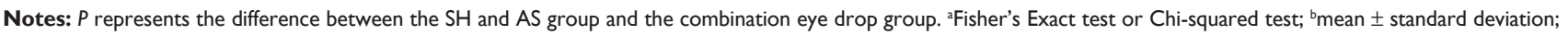

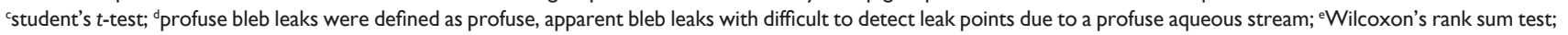
*values during the last examination were significantly lower than before treatment within the same group by Wilcoxon's signed rank test $(P<0.05)$.

Abbreviations: $\mathrm{SH}$, sodium hyaluronate; $\mathrm{AS}$, autologous serum.

last examination of the patient in the sodium hyaluronate and autologous serum group was 23 months, whereas this period ranged from 36 to 61 months for the five patients in the combination eye drop group. All other patients required surgical bleb revisions.

There were no statistically significant differences between the mean intraocular pressures in the groups at the time of detection of bleb leak and at the time of the last examination. At the last examination, the mean intraocular pressure of eyes that did not require bleb revision (one eye in the sodium hyaluronate and autologous serum group and all eyes in the combination eye drops group) was $12.8 \pm 7.1 \mathrm{mmHg}$, and the intraocular pressure of the eyes that required bleb revision (six eyes in the sodium hyaluronate and autologous serum group) was $12.3 \pm 6.6 \mathrm{mmHg}$. This difference was not statistically significant $(P=0.902)$.

The break-up time in the combination eye drop group at the time of detection of bleb leaks was $4.4 \pm 2.1$ (range 2-7) seconds and during the last examination was $13.6 \pm 2.9$ (range 11-18) seconds. This difference in break-up time before and after treatment was significant $(P<0.001)$. Ocular surface staining using the Oxford scale was $>$ grade 1 in all patients. However, while there was a tendency for ocular surface staining to improve after treatment in both groups (as assessed by the Oxford grading scale), this improvement was only significant in the combination eye drop group (Table 1). Epithelial defects in the bleb wall were detected in all patients in both groups. The epithelial defects resolved within 1-2 weeks of the start of treatment, and leakage decreased in all patients. Patients who showed resolution of leakage were able to use the eye drops for the full 4 months. On the other hand, patients who showed continued leakage were unable to use the eye drops for the full 4 months, and epithelial defects recurred more than 2 months after the start of treatment. Multiple microcysts in the bleb surfaces were observed following the start of treatment in all patients. In patients with bleb leakages that resolved with conservative treatment, the number of microcysts decreased as the blebs contracted following bleb resolution (Figures 1 and 2). All five patients in the combination eye drop group had abnormal lid margin abnormality scores (mean $2.8 \pm 0.84$ ) and summed meiboscores (mean $3.6 \pm 0.89$ ), so were diagnosed with obstructive meibomian gland dysfunction. The meibomian glands in the bleb surface contact areas were seen to drop out and/or shorten in all patients (Figure 3 ).

One patient in the combination eye drop group had profuse bleb leakage, which recurred one month after cessation of treatment with combination eye drops (Figure 2). 
Treatment with combination eye drops was recommenced for this patient, and the leak resolved after 5 months. Treatment of this patient with combination eye drops continued for 43 months, after which only the $0.1 \%$ sodium hyaluronate eye drops were administered for the next 14 months. For this patient, the duration from recommencement of combination eye drop therapy to resolution of bleb leakage was considered to be the period between the start of treatment and resolution of the bleb leak.

\section{Discussion}

Usually either conservative treatment or laser therapy is first used to treat late-onset bleb leaks, and surgical revision is performed when these methods prove ineffective. ${ }^{19}$ Conservative treatment of late-onset bleb leaks includes use of therapeutic bandage contact lenses, ${ }^{20}$ autologous blood injections, ${ }^{21,22}$ and autologous serum eye drops. Surgical revision of late-onset filtering blebs attempts to cover the blebs with various membranes, such as the conjunctiva, ${ }^{19}$ amnion, ${ }^{19,23}$ corneal lamellar grafts, ${ }^{24}$ or buccal mucous membranes. ${ }^{25}$ Therefore, the present study attempted to produce a membrane-like substance that could be used to cover the blebs during conservative treatment. We noticed that the tear film, which is similar to a liquid membrane, already covers the blebs. Combined treatment with eyelid massage and warm compresses to improve the lipid layer and sodium hyaluronate and autologous serum eye drops to improve the aqueous layer is very effective in augmenting the tear film. This treatment protects the ocular surface and improves the condition and stability of the tear film. ${ }^{7}$ The treatment used in the present study could be described as covering the defective bleb surface with a well functioning tear film to promote healing and stop aqueous leakage.

A previous study reported that thin and avascular bleb walls with a denuded epithelium and underlying full thickness stromal damage (detected after trabeculectomy with mitomycin C) are likely to be less effective physical barriers against bacteria. ${ }^{3}$ These bleb walls compromise the conjunctival contribution to the ocular innate immune response and have a high risk of complications due to infection. ${ }^{3,4}$ All patients in both groups in the present study showed resolution of epithelial defects in the bleb walls 1-2 weeks after commencement of treatment. Therefore, the risk of bleb infection was lower for patients in this study relative to that of others who suffer from untreated bleb leakages with defects in the bleb walls. However, a relatively long treatment period was necessary to stop the bleb leakage. Therefore, attending physicians should fully explain the risks of bleb infection to patients, and must be prepared to treat bleb infections as soon as possible when they occur.

In this study, we expected to see cumulative effects when we initially treated patients with OSD and late-onset bleb leakages with separate sodium hyaluronate, autologous serum, and ofloxacin eye drops. However, while the epithelial defects temporarily resolved for a few months, this treatment method was not effective for completely resolving leakages in most patients. This may have been due to the difficulty that patients had in adhering to the regimen because of the need to administer high doses of the eye drops frequently. In addition, the cumulative effects of these therapies may not have been sufficient to treat OSD and the bleb leakages. In the one successful case in the sodium hyaluronate and autologous serum group, the bleb leak may have resolved because it was mild and the patient continued instillation of the eye drops until resolution. Therefore, in an attempt to resolve the other cases successfully, we changed the treatment method by combining the drops to decrease the dosage frequency. Moreover, we also examined obstructive meibomian gland dysfunction in the combination eye drop group and treated these patients with eyelid massage and warm compresses. It is very important to maintain tear film if treatment of obstructive meibomian gland dysfunction is to be successful. The lipids that form the lipid layer reduce evaporation of tear fluid ${ }^{26}$ and relieve the friction generated by blinking. ${ }^{27}$

Arita et $\mathrm{al}^{28}$ reported that contact lens wearers show a significantly greater degree of meibomian gland loss than nonwearers. One major cause of this loss is that contact lenses cause chronic irritation of the meibomian glands via the conjunctiva. Moreover, the authors reported that long-term use of anti-glaucoma eye drops promotes meibomian gland loss and could be a reason for the subclinical conjunctival inflammation caused by benzalkonium chloride. ${ }^{29}$ In the present study, all patients had previously been treated with anti-glaucoma eye drops for more than one year. In addition, the meibomian glands were shortened in all patients in the combination eye drop group, particularly in the areas that were in contact with the bleb surface. Therefore, chronic irritation of the meibomian glands by the blebs through the conjunctiva, coupled with inflammation induced by benzalkonium chloride in the anti-glaucoma eye drops, may have contributed to meibomian gland loss.

Break-up time is a test of tear film stability, ${ }^{11}$ and obstructive meibomian gland dysfunction is known to shorten the break-up time. ${ }^{17}$ Break-up time increased after treatment in the combination eye drop group in the present study, suggesting that this method is effective for treating obstructive 
meibomian gland dysfunction and improving the stability of tear film. Microcysts that appear after improvement in conjunctival epithelial defects in the bleb wall are reportedly the result of re-epithelialization, as leakage from the area of defective epithelium is suppressed by the thin conjunctival epithelium. ${ }^{5}$ Microcysts are also frequently observed on well functioning thin bleb surfaces. The present findings support the hypothesis that the number of microcysts decreases in response to the thickening of the bleb wall which occurs when the bleb contracts.

One patient in the combination eye drop group who had profuse leakage required ongoing treatment for more than 4 months. Therefore, we suggest that, instead of prescribing uniform treatment for all patients with late-onset bleb leaks, treatment should be modified according to the extent of leakage, the condition of the blebs, and the severity of OSD. Further studies involving larger patient numbers are needed to investigate the efficacy of the combination therapy described here for the treatment of patients with late-onset bleb leaks.

\section{Disclosure}

The authors report no conflicts of interest in this work.

\section{References}

1. Belyea DA, Dan JA, Stamper RL, Lieberman MF, Spencer WH. Late onset of sequential multifocal bleb leaks after glaucoma filtration surgery with 5-fluorouracil and mitomycin C. Am J Ophthalmol. 1997; 124(1):40-45.

2. Azuara-Blanco A, Katz LJ. Dysfunctional filtering blebs. Surv Ophthalmol. 1998;43(2):93-126.

3. Lehmann OJ, Bunce C, Matheson MM, et al. Risk factors for development of post-trabeculectomy endophthalmitis. $\mathrm{Br} J$ Ophthalmol. 2000;84(12):1349-1353.

4. Katz LJ, Cantor LB, Spaeth GL. Complications of surgery in glaucoma. Early and late bacterial endophthalmitis following glaucoma filtering surgery. Ophthalmology. 1985;92(7):959-963.

5. Sagara H, Iida T, Suzuki K, Fujiwara T, Koizumi H, Yago K. Sodium hyaluronate eye drops prevent late-onset bleb leakage after trabeculectomy with mitomycin C. Eye. 2008;22(4):507-514.

6. Matsuo H, Tomidokoro A, Tomita G, Araie M. Topical application of autologous serum for the treatment of late-onset aqueous oozing or point-leak through filtering bleb. Eye. 2005;19(1):23-28.

7. Pflugfelder SC, Geerling G, Kinoshita S, et al. Management and therapy of dry eye disease: report of the Diagnostic Methodology Subcommittee of the International Dry Eye WorkShop (2007). Ocul Surf. 2007;5(2):163-178.

Clinical Ophthalmology

\section{Publish your work in this journal}

Clinical Ophthalmology is an international, peer-reviewed journal covering all subspecialties within ophthalmology. Key topics include: Optometry; Visual science; Pharmacology and drug therapy in eye diseases; Basic Sciences; Primary and Secondary eye care; Patient Safety and Quality of Care Improvements. This journal is indexed on Submit your manuscript here: http://www.dovepress.com/clinical-ophthalmology-journal
8. Sagara H, Yago K. Dry eye and bleb leaks after trabeculectomy with mitomycin C. Folia Ophthalmol Jpn. 2004;55(1):18-21.

9. Gipson IK. Distribution of mucins at the ocular surface. Exp Eye Res. 2004;78(3):379-388.

10. Mengher LS, Pandher KS, Bron AJ, Davey CC. Effect of sodium hyaluronate $(0.1 \%)$ on break-up time (NIBUT) in patients with dry eyes. Br J Ophthalmol. 1986;70(6):442-447.

11. Lemp MA. Report of the National Eye Institute/Industry workshop on Clinical Trials in Dry Eyes. CLAO J. 1995;21(4):221-232.

12. O'Connor DJ, Tressler CS, Caprioli J. A surgical method to repair leaking filtering blebs. Ophthalmic Surg. 1992;23(5):336-338.

13. Paugh JR, Knapp LL, Martinson JR, Hom MM. Meibomian therapy in problematic contact lens wear. Optom Vis Sci. 1990;67(11):803-806.

14. Olson MC, Korb DR, Greiner JV. Increase in tear film lipid layer thickness following treatment with warm compresses in patients with Meibomian gland dysfunction. Eye Contact Lens. 2003;29(2):96-99.

15. Bron AJ, Evans VE, Smith JA. Grading of corneal and conjunctival staining in the context of other dry eye tests. Cornea. 2003;22(7):640-650.

16. Norn MS. Diagnosis of dry eye. In: Lemp MA, Marqurdt R, editors. The Dry Eye: A Comprehensive Guide. Berlin, Germany: Springer; 1992.

17. Arita R, Itoh K, Maeda S, et al. Proposed diagnostic criteria for obstructive meibomian gland dysfunction. Ophthalmology. 2009; 116(11):2058-2063.

18. Budenz DL, Hoffman K, Zacchei A. Glaucoma filtering bleb dysesthesia. Am J Ophthalmol. 2001;131(5):626-630.

19. Feldman RM, Altaher G. Management of late-onset bleb leaks. Curr Opin Ophthalmol. 2004;15(2):151-154.

20. Blok MD, Kok JH, van Mil C, Greve EL, Kijlstra A. Use of the Megasoft bandage lens for treatment of complications after trabeculectomy. Am J Ophthalmol. 1990;110(3):264-268.

21. Leen MM, Moster MR, Katz LJ, Terebuh AK, Schmidt CM, Spaeth GL. Management of overfiltering and leaking blebs with autologous blood injection. Arch Ophthalmol. 1995;113(8):1050-1055.

22. Dinah C, Bhachech B, Ghosh G. Long-term success with autologous blood injection for leaking trabeculectomy blebs. Br JOphthalmol. 2010; 94(3):392-393.

23. Rauscher FM, Barton K, Budenz DL, Feuer WJ, Tseng SC. Long-term outcomes of amniotic membrane transplantation for repair of leaking glaucoma filtering blebs. Am J Ophthalmol. 2007;143(6):1052-1054.

24. Fukuchi T, Matsuda H, Ueda J, Yamada A, Suda K, Abe H. Corneal lamellar grafting to repair late complications of mitomycin $\mathrm{C}$ trabeculectomy. Clin Ophthalmol. 2010;4:197-202.

25. Rootman DB, Kumar NL, Rootman DS, Trope GE. Buccal mucous membrane for the reconstruction of complicated leaking trabeculectomy blebs. J Glaucoma. 2010;19(4):270-274.

26. Mishima S. Some physiological aspects of the precorneal tear film. Arch Ophthalmol. 1965;73(2):233-241.

27. Bron AJ, Tiffany JM, Gouveia SM, Yokoi N, Voon LW. Functional aspects of the tear film lipid layer. Exp Eye Res. 2004;78(3):347-360.

28. Arita R, Itoh $\mathrm{K}$, Inoue $\mathrm{K}$, Kuchiba A, Yamaguchi T, Amano S. Contact lens wear is associated with decrease of meibomian glands. Ophthalmology. 2009;116(3):379-384.

29. Arita R, Itoh K, Maeda S, et al. Effects of long-term topical antiglaucoma medications on meibomian glands. Graefes Arch Clin Exp Ophthalmol. 2012;250(8):1181-1185. Epub 2012 Feb 18.

\section{Dovepress}

PubMed Central and CAS, and is the official journal of The Society of Clinical Ophthalmology (SCO). The manuscript management system is completely online and includes a very quick and fair peer-review system, which is all easy to use. Visit http://www.dovepress.com/ testimonials.php to read real quotes from published authors. 